$D_{i}(i=1, \cdots, k)$ denoting $D$ the symbol of differentiation, and $D_{i}(i=k+1, \cdots, n)$ denoting $\Delta$ the symbol of differences.

7. The first part of Professor Miller's second paper is devoted to a consideration of all the possible groups which can be generated by two operators $s_{1}, s_{2}$ when these operators satisfy two conditions of the form

$$
s_{1}^{\alpha_{1}} s_{2}^{\alpha_{2}} s_{1}^{\alpha_{3}} \cdots=1, \quad s_{1}^{\beta_{1}} s_{2}^{\beta_{2}} s_{1}^{\beta_{3}} \ldots=1,
$$

where each one of a set of consecutive exponents is unity while each of the other exponents is zero. He proved the following theorem : If identity can be obtained in two ways by forming the continued product with $s_{1}, s_{2}$ taken alternately as factors, then the largest group $G$ generated by $s_{1}, s_{2}$ is of finite order except when the number of factors in both products is even, or when the number of these factors is the same odd number in both products and the same operator occurs an odd number of times in each. When the total number of times that each factor appears in the two products is the same and the number of factors in each product is odd then $G$ is a cyclic group whose order is this total number. Among other theorems proved in this paper is the following: If two operators satisfy the condition $\left(s_{1} s_{2}\right)^{a}=\left(s_{2} s_{1}\right)^{\beta}, \alpha$ and $\beta$ being relatively prime, the order of $s_{1} s_{2}$ may have an arbitrary value $n$, which is prime to $\alpha$ and $\beta$, and hence $s_{1} s_{2}=\left(s_{2} s_{1}\right)^{\gamma}$. The order of $s_{2}$ is an arbitrary multiple of the exponent $e$ to which $\gamma$ belongs modulo $n$ and hence the order of $G$ is a multiple of en. All such groups are solvable.

F. N. CoLE, Secretary.

\title{
THE KÖNIGSBERG MEETING OF THE DEUTSCHE MATHEMATIKER-VEREINIGUNG.
}

The twenty-first annual meeting of the Deutsche Mathematiker-Vereinigung was held at Königsberg, Prussia, September 18-24, 1910, in affiliation with the eighty-second convention of the Society of German naturalists and physicians.

A general reception for all the sections was held at the Festhalle of the Tiergarten on the evening of September 18; the division into sections and the organization of each took place the following morning. Six sessions of the Vereinigung 
were held, under the chairmanship of Professors Hilbert, Meyer, v. Mangoldt, and Schoenflies.

The following papers were read:

(1) R. Fueter, Basel, "Fields of classes of complex multiplication, and its influence on the development of the theory of numbers."

(2) D. Hilbert, Göttingen, "On diophantine differential equations."

(3) A. HAAR, Göttingen, "Illustrations of and additions to the principles explained by Professor Hilbert."

(4) P. Konbe, Göttingen, "Concerning conformal representation of multiply connected regions."

(5) E. Müller, Vienna, "Some groups of theorems regarding oriented circles in the plane."

(6) F. Meyer, Königsberg, "A generalization of the concept of curvature."

(7) H. Liebmann, Leipzig, "On elementary constructions in non-euclidean space."

(8) L. Bieberbach, Göttingen, "On the group of motions in euclidean geometry."

(9) F. ENGEL, Greifswald, "A generalization of the infinitesimal parallel transformation."

(10) E. Papperitz, Freiberg, "On drawing in space" (with the exhibition of apparatus for kinodiaphragmatic projection).

(11) A. Schonnflits, Königsberg, "On the concept of definitions."

(12) A. Witring, Dresden, "Communications concerning some manuscripts of Newton."

(13) W. v. Ignatowski, Berlin, "The principle of relativity."

(14) F. Meyer, Königsberg, "On the theory of rotations."

(15) O. Toeplitz, Göttingen, "Some applications of the theory of an infinite number of variables."

(16) H. WEyL, Göttingen, "Developments in series, and the integral representation of arbitrary functions."

(17) A. Sommerfeld, Munich, "Development of Green's function by means of characteristic functions."

(18) W. Krebs, Grossflotbeck, "New developments in the spectral photography of the sun."

(19) W. KREBS, Grossflotbeck, "Solar activity as volcanic action. Foundations of the theory."

In addition to the preceding papers, five others were read 
before the joint meeting of the sections of mathematics, physics, and geodesy. They were devoted to an estimate of the influence of Bessel and of F. Neumann. The business meeting of the society occupied the entire morning session of September 21 . Professor Schur, of Strassburg, was elected president for the coming year. Professors Dyck, of Munich, and Lilienthal, of Münster, were elected to the Council. The next meeting of the Vereinigung will be held at Carlsruhe in September, 1911.

Abstracts of some of the papers are given below, the numbers corresponding to those in the list above.

1. Kummer recognized that in the theory of algebraic numbers the field of the investigation must be extended until all the properties can be recognized. Apart from the concept of ideals, the Kronecker theory of complex multiplication forms the most important recent extension, since it brings in the aid of another hyperfield in order to develop the theory of numbers of an imaginary quadratic field. Besides the known concepts of field, rings, orders, moduli, it is also necessary to consider regions in which numbers of the set remain such by multiplication and division. Professor Fueter has studied such sets, which he here calls rays. Ideals are divided into classes of rays. If any equation in a field $K$ is given, a definite ray belongs to it, connecting it with the known theory. Equations are made of exponential functions $e^{2 n i z}$, $z$ being rational. If we consider the law of factoring of the prime ideals of $K$ in the hyperfield, we obtain the law of reciprocity. By this means we may calculate the number of classes in the field. For the case in which the ray is the field itself, Hilbert and Furtwängler have constructed the associated equations by arithmetic means.

5. Professor Müller emphasized the importance of cyclographical representation for geometric investigations and gave some illustrations. He obtained, for example, from the theorem : if of the four pairs of tangent planes which can be passed through the sides of a simple quadrilateral to a curve of class 2 , four planes pass through a point, then the remaining ones also pass through a point, by means of cyclographic representation, a theorem regarding directed circles and straight lines, of which a number of theorems of Steiner (Crelle, volume 11 (1831), pages 117-129) are special cases. He showed further that the Feuerbach theorem, dualized in space, becomes: 
if $t_{1}, t_{1}^{\prime} ; t_{2}, t_{2}^{\prime} ; t_{3}, t_{3}^{\prime}$ are pairs of points of a quadratic cone, the lines joining the points of a pair passing through a fixed point, then the planes $\left(t_{1} t_{2} t_{3}^{\prime}\right),\left(t_{1} t_{2}^{\prime} t_{3}\right),\left(t_{1}^{\prime} t_{2} t_{3}\right),\left(t_{1}^{\prime} t_{2}^{\prime} t_{3}^{\prime}\right)$ cut the cone in four curves, touched by a plane section of the surfaces. In this way only can the theorems in the plane express their whole meaning.

9. If the expression $d s=\omega\left(x, y, y^{\prime}\right) d x$ is taken for element of arc, the equation $d s=\delta t$ defines an infinitesimal circle of radius $\delta t$ and center $x, y$. If these circles are constructed for all points of a curve $y=f(x)$, there results, as envelope, an infinitesimally different curve, obtained from the formula by an infinitesimal contact transformation. A particular case is the infinitesimal parallel transformation of the euclidean plane. If now an arbitrary contact transformation is performed upon the element of arc, a new form is obtained,

$$
d s=\left[\alpha\left(x, y, y^{\prime}\right)+\beta\left(x, y, y^{\prime}\right) y^{\prime \prime}\right] d x,
$$

and the contact transformation previously defined is invariant with regard to contact transformations. A direct definition of this transformation is possible, based upon the new form of the element of arc. The purpose of Professor Engel's paper was to supply such a definition.

10. Professor Papperitz discussed the operations of drawing in space, presenting his apparatus for kinodiaphragmatic projection. The apparatus serves the purpose of representing geometric figures by means of projection of movable slits and in fact makes it possible to represent sharply and exactly the most diverse plane and space curves, and to illustrate changes of form produced by changing constants. The images in space are visible from every side. The process is patented; it should materially aid in the instruction in geometry. A prospectus will soon be published by Teubner. The fundamental idea is the following: The three necessary provisions for the representation are 1) adjustable source of light, 2) the original curve, 3) screen; the first is retained, but the others may be replaced by new apparatus, which will actually generate the image. These generators are constructed by mathematical rules, and select rays of light, part being reflected, part absorbed. Illustrations of systems of conics and of curves or quadrics were exhibited. 
12. Dr. Witting reported on the preservation and the condition of the manuscripts of Newton in the library of Cambridge University, and gave some details of unpublished papers on mathematics, relating particularly to early investigations on the calculus.

At the joint meeting Professor Wangerin considered Franz Neumann (1798-1895) first in connection with mathematical physics, in which he was the first and for a long time the ablest German representative; he then discussed his achievements as an academic teacher, and finally as a mathematician.

A publication of 1826 on the Apollonius tactic problem is not well known; while the method is similar to that of French writers of the time, the treatment is more general in that contact is replaced by intersection at given angles, and both cones and spheres are discussed.

There are three purely arithmetic papers on the theory of harmonic functions. In the first (1828), the problem is solved of expanding an unknown function of the latitude and longitude based upon observations into a finite series of Laplace functions. The second (1848) discusses the application of harmonic functions to problems in the theory of the potential on surfaces of revolution, in particular to determine the distribution of terrestrial magnetism. In this paper the Neumann integral first appears. The third is an extensive essay (1878) concerning adjoint spherical harmonics in which the secondary index is larger than the principal index. A method is perfected by which the product of two spherical harmonics can be expanded in a series of harmonic functions. In many other investigations analytical skill of high order is noticeable, particularly in erystallography. Least squares were first applied to harmonize crystallographic measurements. Experimental researches in specific heat were based upon analytic developments (1830-1834). In optics new roads were broken, particularly in double refraction (1832), reflection of metals (1837), color of biaxial crystals in polarized light (1834), and many others, all based on mathematical foundations. Since 1840 most of Neumann's investigations were in connection with electricity.*

\footnotetext{
* See Wangerin: "Franz Neumann, sein Wirken als Forscher und
} Lehrer," Braunschweig, Vieweg, 1907. 